\title{
The role of polymorphisms of thiopurine methyltransferase in therapy with Azathioprine: preliminary study
}

\author{
Francesca Mortillaro, ${ }^{1}$ Carmelo Fabiano, ${ }^{2}$ Maria Piccione, ${ }^{3}$ Marco Giammanco, ${ }^{4}$ Fabio Venturella ${ }^{1}$ \\ ${ }^{1}$ Department of Scientific, Biological, Chemical and Pharmaceutical Tecnologies, University of Palermo; ${ }^{2}$ Laboratory \\ of Molecular Genetics, “Villa Sofia-Cervello" Hospitals; ${ }^{3}$ Medical Genetics, “Villa Sofia-Cervello" Hospitals; \\ ${ }^{4}$ Department of Experimental Biomedicine and Clinical Neurosciences, University of Palermo, Palermo, Italy
}

\begin{abstract}
Azathioprine is an immunosuppressant drug belonging to the class of thiopurines widely used in clinical therapy. Its immunosuppressive action is linked to the substantial action mechanism in the inhibition of the synthesis of nitrogenous bases purine carried out in T-lymphocyte. The level of such medication limit resides in side effects such as myelosuppression and the development of tumours. The occurrence of side effects is linked to the presence of genetic polymorphisms of Thiopurine methyltransferase (TPMT). To date, 40 allelic variants for TPMT have been detected. However, those responsible for the reduction of enzyme activity are three: $* 2, * 3 \mathrm{~A}, * 3 \mathrm{C}$. The presence of one of the three polymorphisms makes the enzyme susceptible to degradation at proteasome level, and exposes the patient to high levels of the active drug that increases the probability of an occurrence of its side effects. Therefore, the Food and Drug Administration imposed the
\end{abstract}

Correspondence: Francesca Mortillaro, Department of Scientific, Biological, Chemical and Pharmaceutical Tecnologies, University of Palermo, via Archirafi 20, 90123 Palermo, Italy.

E-mail: francesca.mortillaro92@gmail.com

Key words: Azathioprine; Thiopurine methyltransferase;

Pharmacogenetics; Polymorphisms of metabolising enzymes drugs.

Contributions: the authors contributed equally.

Conflict of interest: the authors declare no potential conflict of interest.

Funding: none.

Conference presentation: part of this paper was presented at the National SIBS Congress, Trapani, Italy, 27 October 2017.

Received for publication: 24 July 2018.

Revision received: 17 October 2018.

Accepted for publication: 28 October 2018.

CCopyright F. Mortillaro et al., 2018

Licensee PAGEPress, Italy

Journal of Biological Research 2018; 91:7717

doi:10.4081/jbr.2018.7717

This article is distributed under the terms of the Creative Commons Attribution Noncommercial License (by-nc 4.0) which permits any noncommercial use, distribution, and reproduction in any medium, provided the original author(s) and source are credited. execution of a genetic test of TPMT typing in order to determine if the drug therapy is appropriate to the metabolic characteristics of the patient. The study had the aim of identifying the prevalence of the three aforementioned polymorphisms related to TPMT in a sample population in Palermo, Italy, highlighting the differences related to the sex of the patient and highlighting the main phenotypes. The results showed prevalence in the population of the absence of polymorphism. Among the most frequent polymorphisms is the $* 3 \mathrm{~A}(3 \%)$. A percentage of $1.5 \%$ was found for the polymorphism *3C. No polymorphism *2 was identified in the population analyzed.

\section{Introduction}

Pharmacogenetics is the branch of pharmacology that deals with the influence that the genetic component determines in pharmacological response. ${ }^{1-4}$ This discipline revolutionizes the way in framing clinical therapy. Until 1959, a collective approach involving the administration of drugs was used, one size fits all. Pharmacogenetics establishes a new principle of administration: the right dose for the right patient at the right dose and time. The purpose of pharmacogenetics is to identify the drug that suits the patient and determines the therapeutic effect without causing any side effects. ${ }^{5}$

Pharmacogenetics can reach its goals by identifying genetic polymorphisms. For a genetic polymorphism, there is the presence of two or more allelic variants within the same locus, of which the least common one has a frequency greater than $1 \%{ }^{2,6}$ To date, different types of polymorphisms have been recognized: Single Nucleotide Polymorphisms (SNPs); Restriction Fragment Length Polymorphisms (RFLPs); the Variable Number Tandem Repeats (VNTRs); Short Tandem Repeats (STRs); the Copy Number Variations (CNV). ${ }^{6}$ SNPs represent the most common polymorphisms in humans. These are point genetic variations that involve a single position in the gene at which two alternate nucleotides can exist. ${ }^{1,2,6}$ RFLPs are polymorphisms of DNA fragment length produced by cutting with restriction enzymes. These polymorphisms result from the variation of a DNA sequence that produces the loss or formation of a restriction site.

VNTRs are fragments of length ranging from 9 to 100 pairs of bases due to repetitions of repeated sequences in tandem. They are also known as minisatellites.

STRs, also known as microsatellites, consist of a variable number of short sequences, from 1 to 6 pairs of bases, repeated in tandem. ${ }^{6}$

Copy number variations are highlighted by comparing the 
genome of different individuals. This results in variations in the number of copies of a sequence. The affected DNA segment may range from 1 kilobase to several megabases. A bearer of a $\mathrm{CNV}$ may have fewer copies than the two, or a higher number of copies for the same gene. ${ }^{1,6}$

Within pharmacological therapy, genetic polymorphisms may affect both pharmacokinetic and pharmacodynamic factors. In the first case, the polymorphisms interest genes encoding proteins involved in absorption, distribution, metabolism and elimination. In the second case, they concern genes coding for the primary therapeutic target of drugs. ${ }^{1-7}$ In particular in the study, we analyzed the effects of the polymorphisms of an enzyme taking part in metabolism: Tiopurine methyltransferase (TPMT). Such an enzyme deals with the metabolism of an immunosuppressive drug: azathioprine. It is a drug that acts as an antimetabolite for purine, adenine and guanine bases. Its action is carried out by $\mathrm{T}$ lymphocytes. From a chemical point of view it is an imidazole derivative of 6-mercaptopurine, also an immunosuppressive drug. Once administered, azathioprine is converted to 6-mercaptopurine. However, it is preferable to administer azathioprine as it has a better pharmacokinetic profile and greater ability to be captured by cells.

Once administered, azathioprine is converted to 6-mercaptocturine. It undergoes a reaction by hypoxanthine-guanine phosphoribosyltransferase in 6-thio-inosine-5'-monophosphate (6-thioIMP). 6-thio-IMP becomes the substrate of the inositol monophosphate dehydrogenase which transforms it into 6-thioxanthosina-5'-monophosphate. This compound is converted from guanine5 '-monophosphate synthetase into 6-thioguanosine-5'-monophosphate. This product undergoes kinase phosphorylation reactions that determine the formation of two products: i) 6-thioguanosine5 '-diphosphate, which is directed to the purine biosynthesis process where deoxy-6-thioguanosine-5'-triphosphate acts as antimetabolite and inhibits DNA synthesis; ii) 6-thioguanosine-5' triphosphate which is directed in the RNA biosynthesis process.

The metabolism of azathioprine is carried out by two enzymes: xanthine oxidase which determines the formation of thiouric acid, an inactive metabolite; TPMT which transforms 6-mercaptopurine into 6-methylthiopurine, an inactive metabolite.

Azathioprine is used in the treatment of maintaining the state of remission of chronic intestinal inflammatory diseases. The effect of azathioprine is that of an immunomodulator, but also of an agent that saves glucocorticoids. In the case of Crohn's disease, it is used both in maintaining the state of remission of the disease, and also as a second-choice drug in the treatment of fungal disease. In the case of ulcerative colitis, it is used in the treatment of glucocorticoid or refractory forms. It is used in subjects where it is not possible to induce remission through aminosalicylates or in patients with severe pathologies where drugs such as cyclosporin and tacrolimus have been used to induce clinical remission. Azathioprine is used in the prevention of transplant rejection in association with other immunosuppressive drugs. It is used as a second-choice drug in the treatment of immunological diseases such as systemic lupus erythematosus, rheumatoid arthritis, psoriatic arthritis, vasculitis, myasthenia gravis, multiple sclerosis, lymphomas and hemolytic anemia. ${ }^{2}$

The TPMT that deals with the metabolism of azathioprine is subject to genomic polymorphism. To date, 40 allelic variants have been recognized for the enzyme. However, they are responsible for the variability of the pharmacological response in three forms: TPMT*3A (460 G>A and $719 \mathrm{~A}>\mathrm{G}), \mathrm{TPMT}^{*} 3 \mathrm{C}(719 \mathrm{~A}>\mathrm{G})$, and finally $\mathrm{TPMT}^{* 2}(238 \mathrm{G}>\mathrm{C})$ which contributes to a lesser extent. These are polymorphisms that affect coding regions, resulting in the formation of unstable and susceptible enzymatic forms within the proteosome, which ultimately corresponds to reduced enzyme activity. ${ }^{1,2}$ As for the aminoacid sequence: in the case of G238C polymorphism, nucleotide substitution causes substitution at position 80 of alanine with proline; in the case of SNP A719G there is a Tyr240Cys replacement; in the case of SNP G460A there is a substitution of alanine with threonine in position $154 .{ }^{8}$

From a clinical standpoint, reduced activity of the TPMT enzyme results in greater drug activity and greater exposure to toxic effects. For these reasons, genotype analysis tests were introduced. The correlation between genotype and phenotype of these tests is almost $100 \%$. These tests allow to predict the level of enzyme activity. From the phenotypical point of view we can distinguish three categories of subjects: i) slow methylators, homozygous subjects for alleles encoding for non-functional enzymes (es $* 2-* 3 \mathrm{C}$ or $* 3 \mathrm{C}-* 3 \mathrm{C}$ ). The methylation level in this category of patients is reduced or absent. Compared with subjects with normal metabolic activity, these have elevated active drug levels as they have less elimination capacity. Therefore, they are more exposed to undesirable effects. To reduce these risks, the dosage should be modified, giving a quantity equivalent to $1 / 10$ to $1 / 15$ of the standard dose. ii) Intermediate methylators are patients with a heterozygous mutation. In this case, the dosage should be reduced to achieve therapeutic efficacy. iii) Normal methylators are those that exhibit wild type alleles, therefore they are endowed with normal metabolism. These subjects may be treated with thiopuric drugs using standard dosages.

Reduced enzymatic activity of TPMT causes an increase in active drug levels present at the systemic circulatory level, which predisposes the subject to adverse events. Among these most feared are myelosuppression, the onset of cutaneous squamous cell carcinomas, non-Hodgkin lymphomas. Therefore, the Food and Drug Administration envisages performing a TPMT genotype assay for the prior identification of subjects who exhibit reduced activity and thus predisposition to the development of adverse events. 8,9

\section{Materials and Methods}

A 209 patient TPMT genotype test was performed at the Molecular Gene Research Laboratory at the "V. Cervello" Hospital in Palermo. Subjects ranged between the ages of 5-89 years and were sub-divided into 85 males and 124 females.

The method used to carry out the TPMT genotype test involved the implementation of three steps: i) extracting DNA from a peripheral blood sample; ii) execution of Polymerase Chain Reaction (PCR); iii) the analysis of the samples extracted.

DNA extraction was conducted from a peripheral blood sample with EDTA, stored at a temperature of $4^{\circ} \mathrm{C}$. The extraction procedure used was the MagCore ${ }^{\circledR}$ Nucleic Acid Extractor HF16 Plus. To extract the DNA, a $200 \mu$ aliquot from the peripheral blood sample was taken. The DNA Extraction Kit was used: MagCore ${ }^{\circledR}$ Genomic DNA Whole Blood Kit 101. The kit used involves magnetic beads that, by affinity, facilitate DNA extraction. The elution volume selected corresponds to $150 \mu \mathrm{l}$. This device allows to obtain a sample of purity corresponding to O.D. A260 / 280 ratio $1.8 \pm 0.1$.

\section{Polymerase chain reaction}

After obtaining the DNA from the extracted sample it was necessary to submit it to PCR, in order to amplify the fragments of DNA at which the analysis of interest was performed. An Amplification Refractory Mutation System was performed to determine the genotype $\mathrm{G} 238 \mathrm{C}$. In the case of polymorphisms 
G460A and A719G, another procedure is carried out by PCRRFLP (Restriction Fragment Length Polymorphisms). This procedure involves the execution of PCR for amplification of the two fragments of interest and the execution of enzymatic digestion.

The appliance used for PCR is Applied Biosystem 2720 Thermal Cyclers. Four different PCR tubes were set up exclusively for the primers specific to the fragments to be amplified. A single volume mix of $100 \mu \mathrm{l}$ was made up as follows: $50.0 \mu \mathrm{l}$ water; 10.0 $\mu 1$ of specific enzyme buffer; $10.0 \mu \mathrm{MgCl}_{2} 20 \mathrm{mM} ; 2.0 \mu \mathrm{l}$ of 40 mM dNTP; $1.2 \mu 1$ Taq Pol; $20.0 \mu \mathrm{l}$ DNA; $6.0 \mu \mathrm{l}$ of Primers. $23 \mu \mathrm{l}$ of this mix was taken and added in 4 numbered test tubes. The specific primers for each fragment were added separately to the 4 test tubes. For the determination of G238C polymorphism, two PCRs were set up: the test tubes were numbered, in the test tube number 1 the forward and reverse primers specific for the wild type variant were added; in the test tube marked with number 2, the primers related to the mutated form were added. At the end of the PCR, the presence or absence of the amplification product is indicative of the allelic variation the subject is carrying. For the determination of the polymorphism G460A a specimen marked with the number 3 in which the specific primers were added was set up. Likewise, the A719G polymorphism was determined: sample 4 was prepared in which an aliquot of the above mix and the specific primers for the fragment to be amplified were added. The 4 tubes were subjected to PCR using program 62.

\section{Electrophoresis on gel}

Subsequently, an electrophoresis on $2 \%$ agarose gel was performed for two purposes: i) determine the genotype G238C; ii) verify DNA amplification during PCR.

The buffer used for electrophoresis is Tris-Borate-Edta (TBE) $1 \mathrm{x}$. This buffer has a $\mathrm{pH}$ of 8 . In addition, a ethidium bromide fluorescent colorant is added to the buffer. The ethidium bromide allows to highlight the DNA bands thanks to its interlayer action. It is important to point out that DNA that is subjected to electrophoresis cannot be picked up and reused for the denaturing action carried out by the colorant. To this end, a rate to be applied to the electrophoresis is withdrawn at the end of the PCR, and another one is retained for the purpose of performing subsequent procedures. At the rate to be subjected to electrophoresis, a marker is added: bromofenol blue. 4 $\mu 1$ of $0.25 \%$ marker are added in Test 1 and $2 ; 10 \mu 1$ of $0.25 \%$ colorant, which is diluted with water in the ratio $1: 2$, are included in Tests 3 and $4.12 \mu \mathrm{l}$ of each tube was charged into the agarose gel wells and a voltage of $150 \mathrm{mV}$ was applied. The electrophoretic stroke lasted an average of 20-25 minutes.

After the electrophoresis was completed, the gel was observed by means of a transilluminator. A GelDoc-It Imaging System UVP was used. This device is equipped with an UV transilluminator that allows the display of DNA bands. Binding of bands is made possible by the use of ethidium bromide. Ethidium bromide is characterized by an absorption peak at $254 \mathrm{~nm}$ wavelength and an emission peak at $590 \mathrm{~nm}$. The device has a filter for ethidium bromide that blocks UV radiation that is emitted by the gel. The filter is placed below the camera and locks wavelength radiation between 580 and $630 \mathrm{~nm}$. Thanks to VisionWork ${ }^{\circledR}$ LS Software you can capture the images of the gel.

\section{Determination of G238C polymorphism}

The gel analysis, thanks to transilluminator, makes evident DNA bands, and this fact allows to determine the presence of G238C polymorphism. The results that could be observed on the gel are three: i) There is a single well-related DNA band, in which the PCR amplification was produced through wild-type primers. In this case the subject hasn't G238C polymorphism. ii) The presence of a single band in the well where the amplified PCR obtained with the mutated allele primers were loaded. In this case the subject is homozygous for the presence of polymorphism, therefore it will be characterized by a reduced enzymatic activity. iii) There are two bands on the gel, one in the well where the amplified PCR obtained with the wild type primers was loaded, the other for the well at which the amplified PCR was added obtained with the addition of primers for the mutated form. The subject is homozygous for the absence of polymorphism, therefore it will show a 50\% metabolic activity reduction.

A negative result can be highlighted if DNA amplification did not occur. This result can be attributed to an operator error such as the omission of a reagent, or in case of contamination, such as the presence of a PCR inhibitor in the starting mix.

\section{Enzymatic digestion of PCR products for G460A and A719G polymorphisms}

In the case of G460A and A719G polymorphisms, RFLP should therefore be performed by enzymatic digestion, using restriction enzymes.

For the determination of the G460A genotype, a mixture of 7.0 $\mu 1$ of water was prepared; $2.0 \mu \mathrm{l}$ of 3 buffers for the enzyme; $10.0 \mu \mathrm{l}$ of PCR DNA; $1.5 \mu \mathrm{l}$ of Mwo I New England Biolabs Enzyme. The mixture was incubated at $60^{\circ} \mathrm{C}$ for $6 \mathrm{~h}$. This procedure can be implemented by using kettles or by using the Applied Biosystem 2720 Thermal Cyclers by selecting the digestion 60 program. Upon completion of enzymatic digestion, coloration was performed with $4 \mu \mathrm{l}$ of blue bromophenol at $0.25 \%$. The digested product was loaded into a $2 \%$ agarose gel. The buffer used was TBE 1x. Ethidium bromide was added as a fluorescent colorant. A voltage of $150 \mathrm{mV}$ was applied. The electrophoretic run was carried out for 20-25 minute. The gel was subsequently removed, dried and placed inside the UVP GelDoc-It Imaging System dark room. In this way it was possible to observe the DNA bands detected to determine the presence or absence of the G460A polymorphism.

Observable results on the gel are three: i) There are two bands, one of $432 \mathrm{bp}$ and the other of $282 \mathrm{bp}$. In this case the subject is homozygous for the absence of polymorphism. ii) The presence of a single band corresponding to a $714 \mathrm{bp}$ fragment is highlighted on the gel. In this case the subject is homozygous for the presence of polymorphism. iii) There are three bands respectively corresponding to fragments of length of $714 \mathrm{bp}, 432 \mathrm{bp}, 282 \mathrm{bp}$. The subject is heterozygous for the presence of polymorphism.

\section{Identification of genotype A719G}

After performing the PCR and ensuring proper amplification, enzymatic digestion was performed. A mixture of $7.0 \mu$ l of water was prepared; $2.0 \mu \mathrm{l}$ of n. 4 buffer for the enzyme; $10.0 \mu \mathrm{l}$ of PCR DNA; $1.5 \mu$ l of Acc I New England Biolabs ${ }^{\circledR}$ Enzyme. The mixture was incubated at $37^{\circ} \mathrm{C}$ for 6 hours. For the implementation of digestion we used both boilers and PCR thermocyclers, or Applied Biosystem 2720 Thermal Cyclers. In the thermocycler, the digestion 37 program was selected. After completion of digestion, $4 \mu \mathrm{l}$ of $0.25 \%$ staining with blue bromophenol blue was carried out and $12 \mu \mathrm{l}$ of the product was loaded into a well of $2 \%$ agarose gel. Electrophoretic run was performed using $1 \mathrm{x}$ concentration TBE buffer. The fluorescent dye added to the buffer was ethidium bromide. The voltage used to perform the electrophoretic stroke is 150 Mv. The electrophoretic run has a total duration of 20-25 minutes. Upon completion of the electrophoretic run, the DNA bands were 
analyzed on agarose gel with the UVP GelDoc-It Imaging System apparatus. The highlights of the UV transilluminator are as follows: i) A single 373 bp DNA band: in this case it is a homozygous subject for the absence of polymorphism. ii) Three bands of length of $373 \mathrm{bp}, 283 \mathrm{bp}, 90 \mathrm{bp}$. The subject has heterozygous polymorphism. iii) Two bands of length of $283 \mathrm{bp}$ and $90 \mathrm{bp}$. The subject in this case is homozygous for the presence of polymorphism.

\section{Results and Discussion}

The epidemiological study conducted at the Molecular Genetics Laboratory at the V. Cervello Hospital in Palermo recruited 209 patients.

The retrospective observational study involving 200 patients from January 2011 until December 2015 under the determination of the genotype for the TPMT enzyme revealed that the allelic variant is TPMT *1 (191/95.5\%). The highest percentage for the presence of

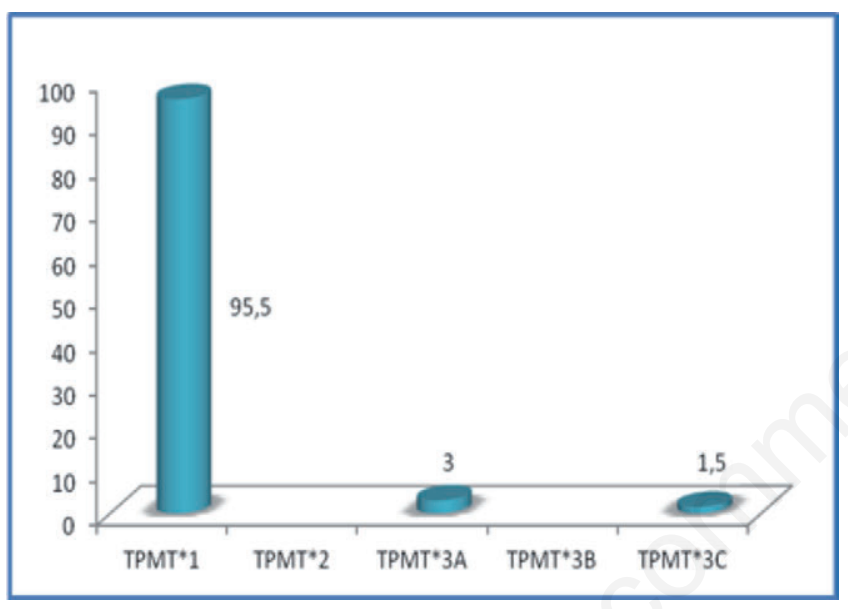

Figure 1. The prevalence of thiopurine methyltransferase (TPMT) polymorphisms from 2011 to 2015.

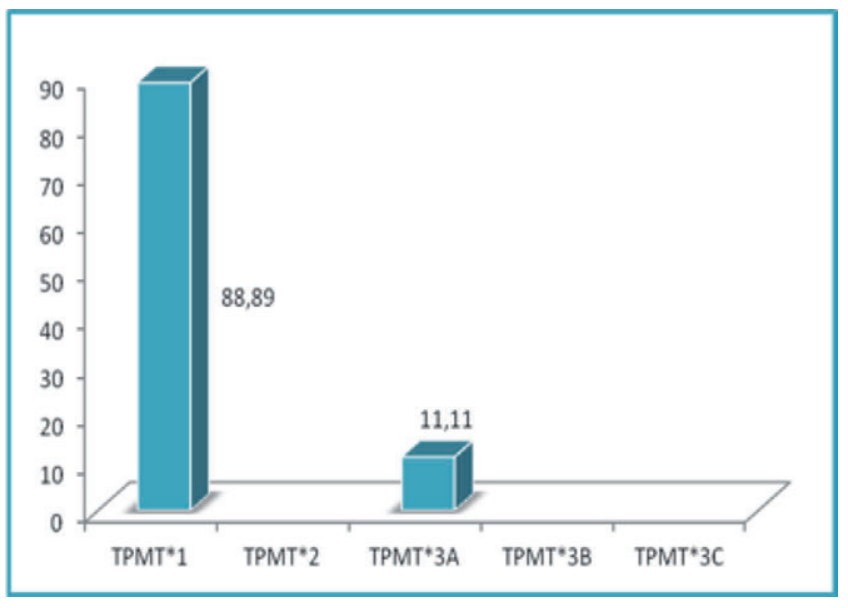

Figure 2. The prevalence of polymorphisms in 2016. polymorphism is that of the *3A form $(6 / 3.0 \%)$. In addition, a small percentage of the form $* 3 \mathrm{C}(3 / 1.5 \%)$ was found (Figure 1$)$.

The study conducted from January to December 2016 enrolled 9 patients. From this study it was found that the most common allelic form is $* 1(8 / 88.89 \%)$. In this study, the only polymorphism found is $* 3 \mathrm{~A}(1 / 11.11 \%)$ (Figure 2$)$.

In addition, an assessment of the prevalence of polymorphisms in the male population ( 85 patients) was performed. A greater frequency of form *1 $(81 / 95.29 \%)$ was found. The only polymorphism found is $* 3 \mathrm{~A}(4 / 4.71 \%)$ (Figure 3$)$.

The frequency of polymorphisms in the female population (124 patients) was evaluated. The highest frequency was found for form *1 (118/95.16\%). Parallel frequency was found for the two allelic variants $* 3 \mathrm{~A}$ and $* 3 \mathrm{C}(3 / 2.42 \%)$ (Figure 4$)$.

Finally, a phenotypic evaluation of the Palermo population sample was conducted. From this the most prevalent phenotype of rapid methylation (199/95.22\%) emerged. There was a prevalence of $2.87 \%$ of intermediate methylators (6 patients) divided as follows: 5 patients were heterozygous for the presence of polymor-

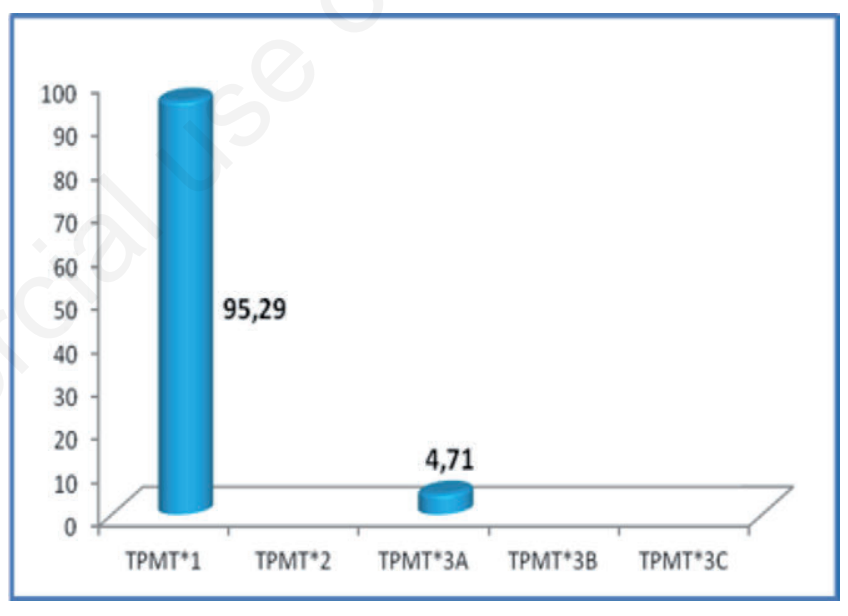

Figure 3. The prevalence of polymorphisms in male population.

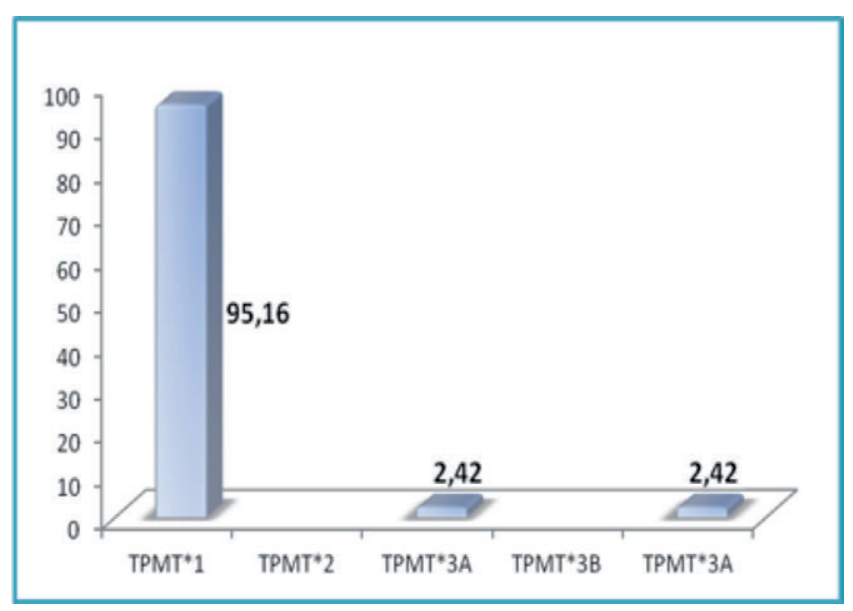

Figure 4. The prevalence of polymorphisms in female population. 
phism *3A $(2.583 \%) ; 1$ patient was found as heterozygous for the presence of polymorphism $* 3 \mathrm{C}(0.287 \%)$. Four patients with slow methylation phenotype $(1.91 \%)$ were found: two patients showed homozygous for polymorphism *3A; two patients with homozygosity for polymorphism $* 3 \mathrm{C}$ (Figure 5 ).

\section{Conclusions}

Widespread use in the pharmacological therapy of azathioprine and the side effects that this drug may induce contribute to understanding the importance of TPMT's genotype assessment. Detection through such analysis of one of the three above-mentioned polymorphisms $(* 2, * 3 \mathrm{~A}, * 3 \mathrm{C})$ results in a reduction in the

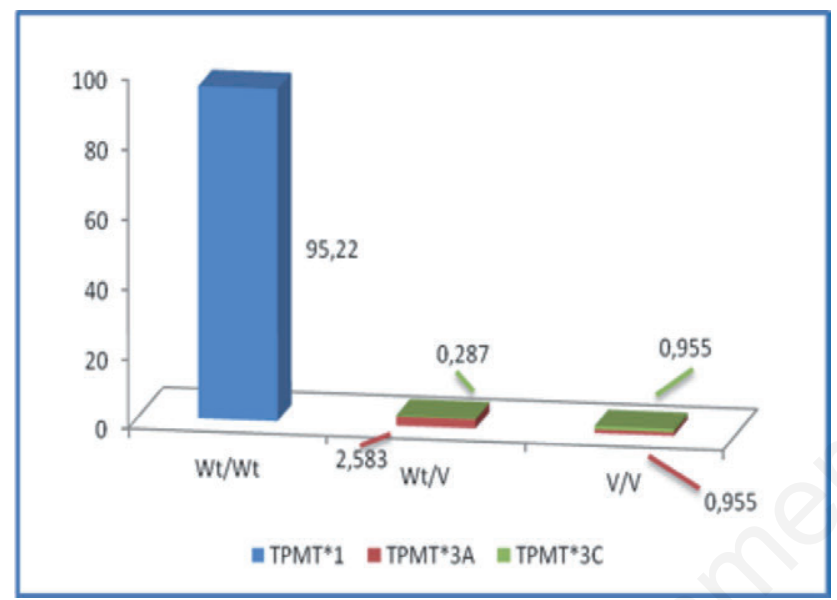

Figure 5. The prevalence of phenotypes in Palermo population. dose of azathioprine and in some cases its replacement with other drugs. This epidemiological study has shown that in the Palermo population the wild type allele is the most present form. Among the polymorphisms in the Palermo population the most common allelic form is that $* 3 \mathrm{~A}$. The allele $* 3 \mathrm{C}$ variant is less frequent, while variant $* 2$ is not detected. All of these data are compliant with the literature in relation to Caucasian subjects. ${ }^{4}$

\section{References}

1. Clementi F, Fumagalli G. Farmacologia generale e molecolare. 4th ed. Torino: UTET; 2012.

2. Annunziato L, Di Renzo GF. Trattato di farmacologia. 2nd ed. Napoli: Idelson-Gnocchi; 2016.

3. Roden DM, George AL. The genetic basis of variability in drug responses. Nat Rev Discov 2002;1:37-44.

4. Serpe L, Canaparo R, Scordo MG, Spina E. Pharmacogenetics of drug-metabolizing enzymes in Italian populations. Drug Metab Pers Ther 2015;30:107-20.

5. Sadée W, Dai Z. Pharmacogenetics/genomics and personalized medicine. Hum Mol Genet 2005;14:R207-14.

6. Torricelli F, Giuliani C. Farmacogenetica: aspetto diagnostici, applicazioni cliniche e prospettive future. Caleidoscopio Italiano, Medical System; 2010.

7. Sim SC, Kacevska M, Ingelman-Sundeberg $M$. Pharmacogenomics of drug-metabolizing enzymes: a recent update on clinical implications and endogenous effects. Pharmacogenomics J 2013; 13: 1-11.

8. Tiopurina S-metiltransferasi (TPMT). Genoma Group. Available from http://www.laboratoriogenoma.eu/eng/analyses_subcategory.asp? IdCat $=31 \&$ IdSubCat $=579$

9. Dean L. Azathioprine therapy and TPMT genotype. In: Pratt V, McLeod H, Rubinstein W, eds. Medical Genetics Summaries. Bethesda, MD, USA: National Center for Biotechnology Information; 2012. 